\title{
Triplet excitons as sensitive spin probes for structure analysis of extended defects in microcrystalline silicon
}

\author{
Christoph Meier, Christian Teutloff, Jan Behrends, * and Robert Bittl \\ Berlin Joint EPR Lab, Fachbereich Physik, Freie Universität Berlin, Arnimallee 14, D-14159 Berlin, Germany \\ Oleksandr Astakhov \\ Forschungszentrum Jülich, Institut für Energie- und Klimaforschung 5-Photovoltaik, D-52425 Jülich, Germany
}

\author{
Klaus Lips \\ Berlin Joint EPR Lab, Institute for Nanospectroscopy, Helmholtz-Zentrum Berlin für Materialien und Energie, \\ Albert-Einstein-Str. 15, D-12489 Berlin
}

(Received 24 March 2016; revised manuscript received 9 June 2016; published 6 July 2016)

\begin{abstract}
Electrically detected magnetic resonance (EDMR) spectroscopy is employed to study the influence of triplet excitons on the photocurrent in state-of-the-art microcrystalline silicon thin-film solar cells. These triplet excitons are used as sensitive spin probes for the investigation of their electronic and nuclear environment in this mixedphase material. According to low-temperature EDMR results obtained from solar cells with different ${ }^{29} \mathrm{Si}$ isotope concentrations between $0.01 \%$ and $50 \%$, the triplet excitons reside at extended defects in the crystallites of microcrystalline silicon that give rise to shallow states in the silicon band gap. The excitons possess a rather delocalized wave function, couple to electron spins in conduction band tail states nearby, and take part in a spin-dependent recombination process. Our study shows that extended defects such as grain boundaries or stacking faults in the crystalline part of the material act as charge carrier traps that can influence the material conductivity.
\end{abstract}

DOI: 10.1103/PhysRevB.94.045302

\section{INTRODUCTION}

Triplet excitons play an important role for the optical and electrical properties of semiconductors and semiconductor devices. For instance, the spin degree of freedom can decisively influence the free charge carrier generation rate in photovoltaic devices based on disordered organic semiconductors [1-3]. Here, the formation of triplet excitons is a crucial step that can limit the free charge carrier yield and thus the efficiency of the solar cells. Presently, the role of the triplet states in loss mechanisms is being actively discussed [2,4]. Triplet excitons are also involved in photochemical up-conversion [5] and down-conversion [6] processes that can potentially increase the efficiencies of both organic and inorganic solar cells beyond the Shockley-Queisser limit [7].

In pure inorganic semiconductor crystals, structural and point defects can lead to localized excitations. Triplet states were previously identified in crystalline silicon (c-Si) using electron paramagnetic resonance (EPR) spectroscopy [8]. Electrically detected magnetic resonance (EDMR) measurements have shown that they influence the photoconductivity in this material $[9,10]$.

The fact that triplet excitons were found to substantially influence the electrical and optical properties of both disordered organic semiconductors as well as crystalline inorganic semiconductors suggests that they may also be present in noncrystalline silicon films. An important representative of this material class is hydrogenated amorphous silicon (a-Si:H), which is used as absorber material in thin-film solar cells and

\footnotetext{
*j.behrends@fu-berlin.de
}

has well-studied electrical and optical properties [11]. There is indeed a number of studies reporting triplet exciton signals in photoluminescence spectra of a-Si:H $[12,13]$. The triplet states are identified by means of their several milliseconds lifetime at room temperature. However, indications for the presence of triplet states in thin-film silicon from EPR-based measurements are rather scarce. A broad line was observed in the EDMR spectra of undoped a-Si:H [14]. This signal was attributed to excitonic states affecting the photoconductivity of a-Si:H. Optically detected magnetic resonance (ODMR) studies of porous silicon and siloxene revealed radiative triplet excitons responsible for the strong photoluminescence in these materials [15]. Spin-dependent processes arising from strongly coupled charge-carrier pairs were also found in silicon-rich hydrogenated amorphous silicon-nitride [16].

Hydrogenated microcrystalline silicon $(\mu \mathrm{c}-\mathrm{Si}: \mathrm{H})$ is another thin-film silicon material exhibiting a different morphology than a-Si:H. It is a heterogeneous material that consists of small crystallites mainly in columnar structure that are embedded in an amorphous tissue. $\mu \mathrm{c}-\mathrm{Si}: \mathrm{H}$ is routinely deposited in a plasma enhanced chemical vapor deposition (PECVD) reactor using high hydrogen dilution. Both thin-film silicon variants can thus be conveniently combined in tandem solar cells $[17,18]$ that are fabricated in one and the same deposition system. Since the band gaps of a-Si:H and $\mu \mathrm{c}-\mathrm{Si}: \mathrm{H}$ differ substantially, a combination of both materials in one device allows an efficient use of the solar spectrum and has led to tandem solar cells with efficiencies in excess of $12 \%$ $[19,20]$. Triplet excitons can also be found in $\mu \mathrm{c}-\mathrm{Si}: \mathrm{H}$. ODMR measurements could show that triplet excitons in $\mu \mathrm{c}-\mathrm{Si}: \mathrm{H}$ films affect the optical properties $[21,22]$. In contrast, the influence of triplet excitons on the electrical properties of $\mu \mathrm{c}-\mathrm{Si}: \mathrm{H}$ is 
not clear yet. On one hand, one could assume that triplets in the amorphous regions contribute to the charge transport in a similar way as in pure a-Si:H. On the other hand, the crystalline regions can possibly accommodate triplet excitons as well, affecting the electrical properties through similar processes as in crystalline silicon. EDMR-based techniques can directly probe the influence of triplet excitons on the charge transport. They can thus be applied to answer the questions regarding the origin of the triplet exciton (TE) states, how they participate in electronic transport of the solar cell at low $T$, where the TE states are located in the mixed-phase material, and how they correlate with other known defects such as dangling bonds (db) and conduction band tail (CE) states.

In this study we use different pulsed (p) EDMR methods like field-swept experiments at microwave (mw) frequencies $v_{0}=3.5 \mathrm{GHz}$ ( $S$ band) and $9.8 \mathrm{GHz}$ ( $X$ band), Rabi nutation measurements, or electrically detected electron spin echo envelope modulation (ED-ESEEM) experiments at $T=5 \mathrm{~K}$ to distinguish triplet signals from other current-influencing processes. Based on this, we clearly identify triplet excitons in $\mu \mathrm{c}-\mathrm{Si}: \mathrm{H}$. Furthermore, field-swept and ESEEM measurements are employed to probe the coupling between the electronic triplet states and nuclear spins in their vicinity. For this purpose we varied the concentrations of the nuclear spin-carrying isotope ${ }^{29} \mathrm{Si}$ in the solar cells. We conclude that the triplet states are located in hydrogen-depleted crystalline regions and couple only weakly to neighboring ${ }^{29} \mathrm{Si}$ nuclei. The results of electrically detected electron electron double resonance (ED-ELDOR) experiments indicate that a triplet exciton (spin quantum number $S=1$ ) has the possibility to couple to a localized electron in a CE state $(S=1 / 2)$ nearby, which might have impact on the current-influencing processes through involvement of three-spin complexes. Finally, we assign the triplet excitons and the spins in CE states to extended defects like grain boundaries and stacking faults in the crystalline parts and suggest a mutual charge carrier transport process involving both the triplet exciton and a CE spin.

Although the transport processes via localized states in the solar cell cannot be directly transferred from $T=5 \mathrm{~K}$ to room temperature, the gained knowledge about extended defects as traps for charge carriers can help to understand recombination processes even at solar-cell working conditions. It is conceivable that triplet excitons in $\mu \mathrm{c}-\mathrm{Si}: \mathrm{H}$ influence the room-temperature charge transport as well. However, the triplet exciton lifetime at elevated temperatures may be too short for EDMR-based detection.

\section{MATERIALS AND METHODS}

\section{A. Samples}

Three types of $\mu \mathrm{c}-\mathrm{Si}: \mathrm{H}$ thin-film solar cells in an EDMRcompatible geometry were deposited on a quartz glass substrate (Corning 1737) in a p-i-n layer configuration by PECVD [18]. Optimized standard deposition conditions [23] were employed. The samples consist of a stack of $800 \mathrm{~nm}$ texture-etched aluminum-doped $\mathrm{ZnO}$ ( $\mathrm{ZnO}: \mathrm{Al})$, boron-doped p- $\mu \mathrm{c}-\mathrm{Si}: \mathrm{H}, \approx 1 \mu \mathrm{m}$ intrinsic $\mu \mathrm{c}-\mathrm{Si}: \mathrm{H}$ absorber, phosphorusdoped n- $\mu \mathrm{c}-\mathrm{Si}: \mathrm{H}$, and $\mathrm{ZnO}: \mathrm{Al}$ with silver as a back contact as shown in Fig. 1(b).

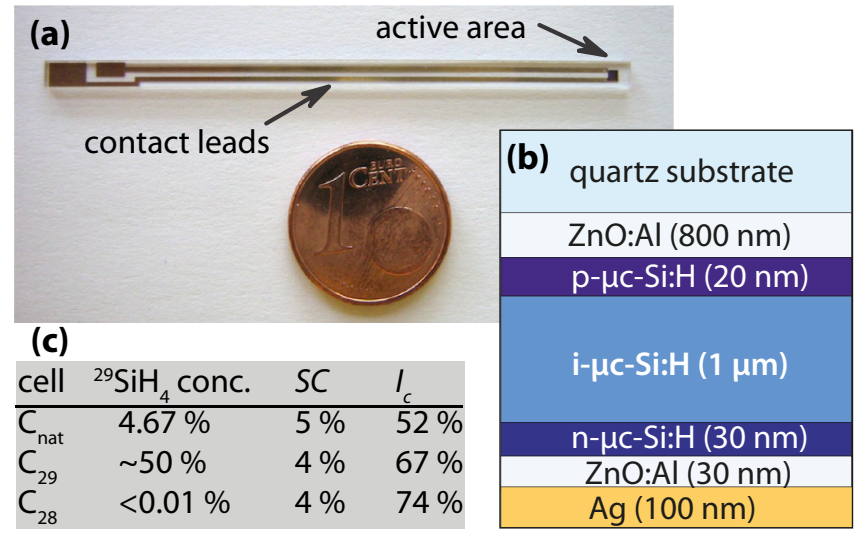

FIG. 1. (a) Photograph of a thin-film solar cell with quartz substrate, conduction lines, and contact pads (left) and the black active cell area (right). (b) Layer structure of the $\mu \mathrm{c}-\mathrm{Si}: \mathrm{H}$ thin-film solar cell. The ${ }^{29} \mathrm{Si}$ abundance of the $\mathrm{i}-\mu \mathrm{c}-\mathrm{Si}: \mathrm{H}$ layer was altered in the three samples. Illumination of the cells takes place through the quartz substrate. (c) Table of the most relevant parameters of the sample deposition: the ${ }^{29} \mathrm{SiH}_{4}$ silane concentration in the silane gas for the intrinsic layer deposition, the silane concentration $\mathrm{SC}=\mathrm{SiH}_{4} /\left(\mathrm{SiH}_{4}+\mathrm{H}_{2}\right)$ within the deposition gas, and the obtained crystalline $\mathrm{Si}$ volume fraction $I_{\mathrm{c}}$ of the $\mu \mathrm{c}-\mathrm{Si}: \mathrm{H}$ material as determined from Raman measurements.

The cells have different ${ }^{29} \mathrm{Si}$ concentrations in the $\mu \mathrm{c}$ $\mathrm{Si}: \mathrm{H}$ intrinsic layer. The first cell $\left(\mathrm{C}_{\mathrm{nat}}\right)$ exhibits the natural abundance of silicon isotopes with $4.67 \%$ of the spin-carrying isotope ${ }^{29} \mathrm{Si}$. The concentration of ${ }^{29} \mathrm{Si}$ was increased in the second cell $\left(\mathrm{C}_{29}\right)$ to $\approx 50 \%$ for the investigation of the electron wave function in the silicon lattice. The third cell $\left(\mathrm{C}_{28}\right)$ was deposited without ${ }^{29} \mathrm{Si}$ nuclei in the intrinsic layer by using high purity silane ${ }^{28} \mathrm{SiH}_{4}(>99.9 \%)$. Silane with a natural isotope abundance was used for the $\approx 30 \mathrm{~nm}$ thick $n$ and $p$-doped layers. The most relevant deposition and film parameters are given in Fig. 1(c). All three cells were laser patterned in order to form a $1 \times 1 \mathrm{~mm}^{2}$ active area solar cell with contact leads for EDMR experiments [cf. Fig. 1(a)].

\section{B. EDMR background}

General requirement for the observation of EDMR is the presence of unpaired spins in the material that are both accessible for EPR manipulation and are involved in conductivity-influencing charge carrier transport processes. Such processes are spin dependent if the spin symmetry of two interacting spins has impact on the process rate [24-26]. Manipulating the spin symmetry by EPR gives rise to a change of the process rates and, thus, to the EDMR current response.

Most signals found in EDMR studies of different devices are due to spin-dependent processes involving weakly coupled pairs of $S=1 / 2$ spins like, e.g., the charge-carrier recombination between ${ }^{31} \mathrm{P}$ donors and $\mathrm{P}_{\mathrm{b} 0}$ states at the $\mathrm{Si} / \mathrm{SiO}_{2}$ interface [27] or hopping via conduction band tail states in a-Si:H $[28,29]$. In this weak coupling regime the spin pair eigenbase consists of the product states of both spins. Thus selective spin flips of one pair spin by a mw $\pi$ pulse converts the surplus of long-lived triplet $\left|T_{+}\right\rangle=|\uparrow \uparrow\rangle$ and $\left|T_{-}\right\rangle=|\downarrow \downarrow\rangle$ pairs 

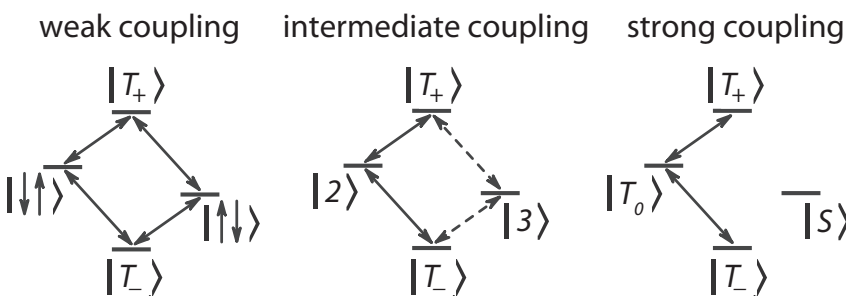

FIG. 2. Sketches showing the four pair states of two coupled spins for negligible, intermediate, and strong spin-spin interaction (from left to right). Allowed EPR transitions are shown by arrows, where solid and dashed arrows indicate high and low transition probabilities. The vertical shift of the energy levels within the sketches is only qualitative.

in the sample to mixed $|\downarrow \uparrow\rangle=\frac{1}{\sqrt{2}}\left(\left|T_{0}\right\rangle-|S\rangle\right)$ and $|\uparrow \downarrow\rangle=$ $\frac{1}{\sqrt{2}}\left(|S\rangle+\left|T_{0}\right\rangle\right)$ pairs (cf. Fig. 2), that are assumed to have a shorter lifetime due to the singlet $|S\rangle$ content. This leads to a higher probability of the subsequent hopping or recombination steps resulting in a change of device conductivity [26,30].

However, when the spin pair is strongly coupled, spin manipulation drives transitions between the states $\left|T_{+}\right\rangle,\left|T_{0}\right\rangle$ and $\left|T_{-}\right\rangle$of the $S=1$ manifold only, whereas the population of the $|S\rangle$ state in the $S=0$ manifold remains unaltered (cf. Fig. 2). This is the reason why signals of strongly coupled spin pairs in principle should not be detectable with EDMR. Only if intersystem crossing (ISC) [31] from the triplet to the singlet manifold is made possible by, e.g., spin-orbit coupling (SOC) $[32,33]$ or if a third spin is involved in the spin-dependent process [34], singlet pair population is generated and the induced current change can be observed as an EDMR signal.

\section{Experiments and data evaluation}

pEDMR measurements were carried out on Bruker spectrometers with working frequencies of $3.5 \mathrm{GHz}$ ( $S$ band) and $9.8 \mathrm{GHz}$ ( $X$ band) corresponding to resonant magnetic fields of $B_{0} \approx 125$ and $350 \mathrm{mT}$ at $g=2$. At both frequencies resonant probe heads ER4118S-MS5 and EN4118X-MD4 were used. Prior to the measurements the solar cells [Fig. 1(a)] were glued on a sample holder and the Ag contact lines on the glass substrate were connected to thin coaxial cables for current detection. This sample holder was mounted in the spectrometer such that the cells were placed in the center of the mw resonator. High microwave power for pulsed EDMR experiments reaching $P_{\mathrm{mw}}=40 \mathrm{~W}$ at $3.5 \mathrm{GHz}$ (solid state amplifier) and $P_{\mathrm{mw}}=1 \mathrm{~kW}$ at $9.8 \mathrm{GHz}$ (traveling wave tube amplifier) were used.

All EDMR experiments were performed at $T=5 \mathrm{~K}$. The sample cooling was accomplished with helium flow cryostats (CF935, Oxford Instruments) controlled by 321 Autotuning (LakeShore) and ITC503 (Oxford Instruments) temperature controllers at $S$ - and $X$-band frequencies, respectively. The continuous illumination of the samples utilizing a DC-driven halogen cold light source from Polytec $(P=150 \mathrm{~W})$ was realized at $3.5 \mathrm{GHz}$ via an optical fiber with a microprism at the top to reflect the light directly onto the $1 \mathrm{~mm}^{2}$ small active cell area and at $9.8 \mathrm{GHz}$ via shining light directly through the optical window into the resonator resulting in photocurrents
$I_{\mathrm{ph}}$ of $\approx 1-20 \mu \mathrm{A}$ at $5 \mathrm{~K}$. This corresponds to current densities in the range of $0.1-2 \mathrm{~mA} \mathrm{~cm}^{-2}$. The photoconductivity of the solar cells was detected with a combined voltage source and current amplifier (Elektronik Manufaktur Mahlsdorf) [35]. For the EDMR experiments the solar cells were operated in reverse bias $(U=-1 \mathrm{~V})$ in order to maximize the carrier collection yield. Low temperature I-V characteristics of the $\mu \mathrm{c}-\mathrm{Si}: \mathrm{H}$ thin-film solar cells and additional information about technical aspects can be found in Ref. [35].

\section{Field-swept EDMR experiment}

EDMR spectra at $S$ and $X$ band were measured for the thin-film Si solar cells by monitoring the current change $\Delta I(t)$ after a mw $\pi$-pulse as function of the magnetic field $B_{0}$ [36]. A pulse length of $t_{\mathrm{p}}=300 \mathrm{~ns}$ was necessary to reduce the spectral broadening by pulse bandwidth effects. The current signal $\Delta I(t)$ reached values up to $\approx 20 \mathrm{nA}$ after the pulse corresponding to a maximum relative current change $\frac{\Delta I}{I_{\mathrm{ph}}}$ of approximately $10^{-3}$.

\section{Electrically detected Rabi nutations}

In the Rabi nutation experiment performed at a constant magnetic field $B_{0}$ the sample is exposed to a mw pulse, whose length $t_{\text {Rabi }}$ is incremented corresponding to an increase of the pulse flip angle $\phi_{\text {Rabi }}$. Measuring the time integral $\Delta Q$ of the EDMR current transient $\Delta I(t)$ as function of $t_{\text {Rabi }}$ yields the transient nutation of the detected electron spins $[37,38]$. The nonmodulating background of a Rabi trace is removed for data evaluation yielding the modulated part. Afterwards, the time trace is apodized by a Hamming window to suppress side bands before fast-Fourier transformation (FFT).

The Rabi frequency $\Omega$ measured under resonance condition is indicative of the electron spin state when the mw $B_{1}$ field is known, since

$$
\Omega\left(m_{\mathrm{S}}, m_{\mathrm{S}}+1\right)=\gamma_{\mathrm{i}} B_{1} \sqrt{S(S+1)-m_{\mathrm{S}}\left(m_{\mathrm{S}}+1\right)}
$$

for an allowed EPR transition involving the substates $m_{\mathrm{S}}$ and $m_{\mathrm{S}}+1$ [39]. Here, $\gamma_{\mathrm{i}}=\frac{2 \pi g_{\mathrm{i}} \beta_{\mathrm{e}}}{h}$ is the gyromagnetic ratio calculated for a spin of species $i$ with $g$ value $g_{i}$, Bohr magneton $\beta_{\mathrm{e}}$, and the Planck constant $h$.

\section{Electrically detected ESEEM}

Two different ESEEM methods based on the two-pulse ( 2 pESEEM) and three-pulse ( 3 pESEEM) echo sequences were employed in this study. Both are routinely used in EPR $[39,40]$. These sequences excite nuclear coherences, which are measured via the detection of the electron spin echo amplitude as function of the interpulse delays. To adapt these methods for EDMR the pulse sequences are augmented by a $\pi / 2$ readout pulse at the time of echo formation to transfer the electron coherence to polarization [cf. Figs. 3(b) and 3(c)] [41]. Hence, the integrated transient current response $\Delta Q$ is measured to observe the echo modulation when nuclear coherences occur. Such an ESEEM effect is observed when $S$ and $I$ are coupled via an anisotropic hyperfine (HF) interaction. Under these conditions otherwise forbidden transitions become weakly allowed, so that the echo amplitude in spin echo experiments 
(a) energy diagram

(b) pEDMR 2pESEEM

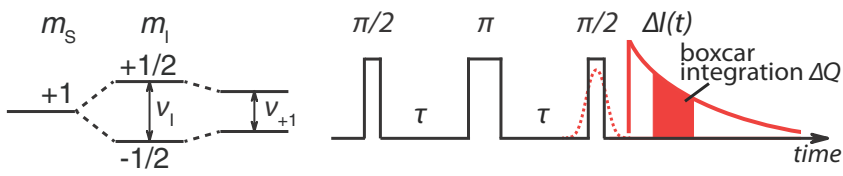

(c) pEDMR 3pESEEM
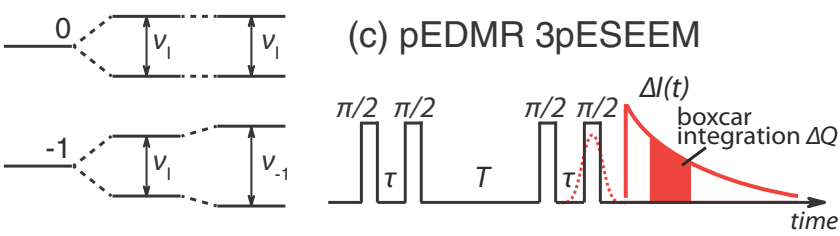

(d) EDELDOR
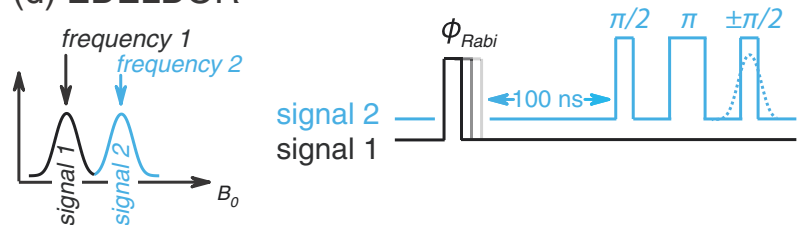

signal 1

FIG. 3. (a) The energy level diagram of an electron spin $S=1$ coupled to a ${ }^{29} \mathrm{Si}$ nucleus $I=1 / 2$ (nuclear $g$ value $g_{\mathrm{n}}=-1.1106$ ). $v_{\mathrm{I}}$ is the nuclear Zeeman frequency, $v_{ \pm 1}$ are the nuclear transition frequencies including the HF interaction. (b) and (c) show the pulse sequences of the EDMR two-pulse and three-pulse ESEEM experiments. (d) ED-ELDOR experiment. Left: scheme of a spectrum with two spin species giving rise to EDMR signals at different $B_{0}$ positions. Right: pulse sequences of the ED-ELDOR experiment for the manipulation of signal 1 and 2 at different mw frequencies.

is modulated by nuclear frequencies. For an $S=1$ system coupling to a nuclear spin with $I=1 / 2$ these frequencies are $v_{ \pm 1}=\sqrt{\left(a \pm v_{\mathrm{I}}\right)^{2}+b^{2}}$ and $v_{\mathrm{I}}$ for the three $m_{\mathrm{S}}$ sublevels $m_{\mathrm{S}}=$ $\pm 1,0$ [cf. Fig. 3(a)]. Here, $v_{\mathrm{I}}$ is the nuclear Larmor frequency and $a, b$ are the secular and pseudosecular part of the HF interaction which are given by $a=A_{\text {iso }}+A_{\text {dip }}\left(3 \cos ^{2} \theta-1\right)$ and $b=3 A_{\text {dip }} \sin \theta \cos \theta$ [39]. $A_{\text {iso }}$ and $A_{\text {dip }}$ are the isotropic and anisotropic HF interaction and $\theta$ describes the angle between external magnetic field vector and the axis connecting the electron and nuclear spins.

The 2pESEEM experiment for EDMR is based on the sequence $\pi / 2-\tau-\pi-\tau-\pi / 2$ as shown in Fig. 3(b) and yields traces with nuclear frequencies $v_{\mathrm{I}}, v_{ \pm 1}$ as well as their sums and differences $v_{\mathrm{I}} \pm v_{ \pm 1}$. Since the echo decay of 2 pESEEM is limited by the electron spin phase memory time $T_{\mathrm{M}}$, the frequency spectrum is artificially broadened. In contrast, the 3pESEEM traces, showing nuclear frequencies $v_{\mathrm{I}}$, $v_{ \pm 1}$, decay with the often longer spin-lattice relaxation time $T_{1}$, which results in a gain in spectral resolution. However, the pulse sequence $\pi / 2-\tau-\pi / 2-T-\pi / 2-\tau-\pi / 2$ [Fig. 3(c)] generates so-called blind spots, which suppress spectral frequencies, whenever $v_{i} \cdot \tau$ is a multiple of 1 . For that reason a $\tau$ value of $80 \mathrm{~ns}$ was chosen to shift the first blind spot in the spectrum to $>10 \mathrm{MHz}$.

The electron nuclear double resonance (ENDOR) experiment is an alternative approach for measuring HF couplings as recently shown in combination with EDMR $[42,43]$. However, the use of radio frequency ENDOR pulses causes strong interference signals in the current detection circuit of the solar cell, which makes the extraction of the pure HF spectra technically very challenging. Electrically detected ENDOR experiments could so far be realized only on the model system of phosphorus donors in $\mathrm{c}-\mathrm{Si}$ with rather strong hyperfine coupling.

The ESEEM experiments were carried out at $X$-band frequencies with high power pulses yielding pulse lengths of $t_{\pi / 2}=10 \mathrm{~ns}$ and $t_{\pi}=20 \mathrm{~ns}$, which were found with Rabi nutation measurements. Phase cycling with two and eight steps was employed in the 2pESEEM and 3pESEEM experiments, respectively, to remove background signals and unwanted echoes from the time traces, as commonly done in EPR [39]. All ESEEM time traces were post processed by (1) removing the unmodulated background, (2) apodizing the remaining modulating part with a ninth order Kaiser window function to suppress frequency side bands, and (3) calculating the frequency spectra by fast-Fourier transformation (FFT).

\section{Electrically detected ELDOR}

The ED-ELDOR experiment [10,44] was used to study the interaction between electron spins. ED-ELDOR detects qualitatively if an electron spin is coupled to another electron spin in the environment, whereas the ELDOR experiment common in EPR quantitatively measures dipolar couplings between interacting electron spins [45].

In the constant-field ED-ELDOR experiment pulses of different mw frequencies are used to selectively excite transitions of both coupled spin species. Rabi experiments are performed on the EPR transition of the first species. If a coupling exists, this Rabi signature is imprinted on the otherwise independent signal amplitude of the second species. Thus, the ELDOR experiment consists of two parts as sketched in Fig. 3(d): First, a Rabi nutation experiment with a stepwise incrementation of the mw pulse flipping angle $\phi_{\text {Rabi }}$ is carried out addressing signal 1 using mw frequency 1 . Second, the echo peak intensity of signal 2 after the EDMR spin-echo pulse sequence $\pi / 2-\tau-\pi-\tau- \pm \pi / 2$ at mw frequency 2 is detected by boxcar integration of the transient current response $\Delta I(t)$. The mw power for the echo sequence was chosen such that a $\pi$-pulse length of $20 \mathrm{~ns}$ was achieved in all experiments. Application of two-step phase cycling within the echo sequence (indicated by $\pm \pi / 2$ ) allows us to detect only the current response due to the spin echo of signal 2 . In addition, ED-ELDOR experiments with different mw power attenuations for the Rabi pulse were performed yielding a change of the signal 1 nutation frequencies. With this the reliability of the results is checked.

\section{Data evaluation}

The data evaluation throughout this study comprising fitting routines, data post-processing, as well as data transformation was done with the help of MATLAB 2012a (The MathWorks, Inc., Natick, MA, USA). The EPR toolbox easyspin [46] implemented in MATLAB was used for the simulation of EPR spectra. 


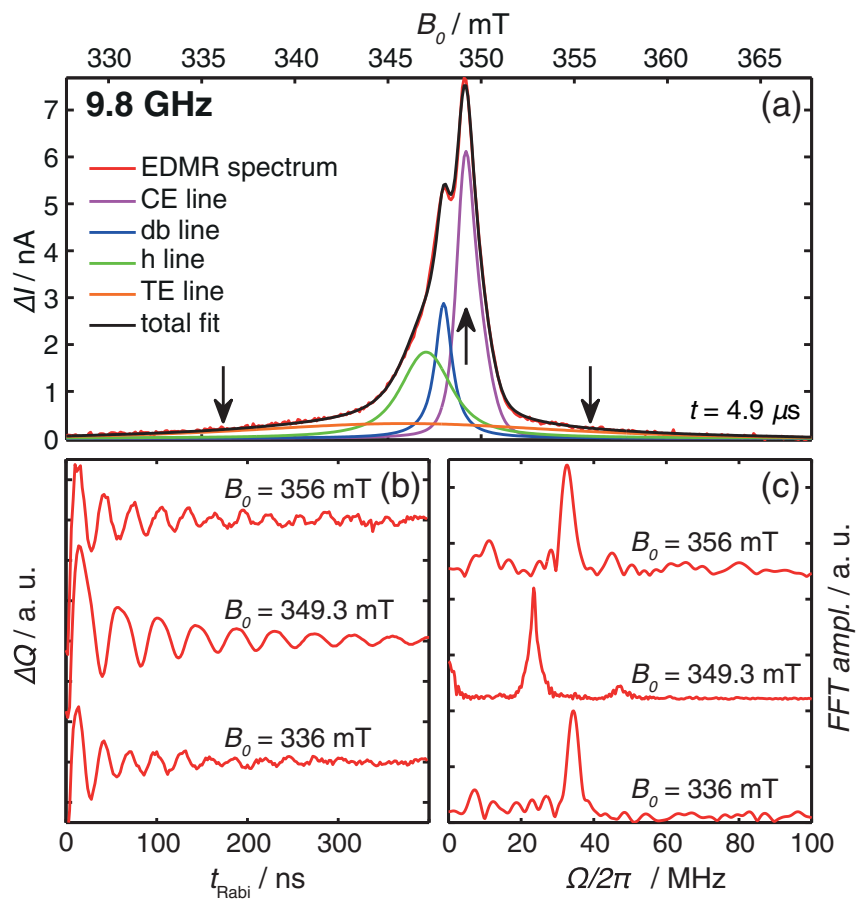

FIG. 4. (a) Field-swept $X$-band EDMR spectrum at $t=4.9 \mu \mathrm{s}$ after the mw pulse together with the fit results. Field positions for Rabi nutation experiments are indicated by arrows. (b) Modulating part of the Rabi traces measured at these three field positions and (c) their corresponding frequency spectra after FFT. Rabi traces and frequency spectra were vertically shifted for clarity.

\section{RESULTS}

\section{A. Field-swept EDMR at $X$ band}

In a first step, the EDMR spectrum of the $\mu \mathrm{c}-\mathrm{Si}: \mathrm{H}$ solar cell $\mathrm{C}_{\text {nat }}$ with natural $\mathrm{Si}$ isotope abundance was measured at $\nu_{0}=$ $9.8 \mathrm{GHz}$ ( $X$ band) at $T=5 \mathrm{~K}$. The spectrum is deconvolved into individual lines and assigned to defects in the material.

The EDMR spectrum obtained at $t=4.9 \mu \mathrm{s}$ after the mw pulse is shown in Fig. 4(a). It consists of several strongly overlapping EDMR signals, which can be decomposed by using their individual signal dynamics [47,48]. Therefore, spectra at different times after the mw pulse are fitted with a set of four lines. This set describes the spectra at all times consistently, because the current dynamics influences only the signal intensity but not the line shapes and positions.

The characteristic $g$ values and line widths of the four resonances are given in Table I. Based on these parameters, three lines can already be associated with paramagnetic centers

TABLE I. The line parameters of the four signals deduced from the $X$ band pEDMR data sets of the $\mu \mathrm{c}-\mathrm{Si}: \mathrm{H}$ solar cell $\mathrm{C}_{\mathrm{nat}}$. For detailed information see Sec. III A.

\begin{tabular}{lccr}
\hline \hline Assignment & g value & Voigtian shape & Line width $\Delta B$ \\
\hline h center & $2.0105(5)$ & {$[1.572 .48] \mathrm{mT}$} & $3.3(1) \mathrm{mT}$ \\
db center & $2.0046(3)$ & {$[0.071 .07] \mathrm{mT}$} & $1.1(1) \mathrm{mT}$ \\
CE center & $1.9978(3)$ & asym. shape $[48]$ & $1.4(1) \mathrm{mT}$ \\
TE center & $2.017(1)$ & {$[11.411 .4] \mathrm{mT}$} & $19(1) \mathrm{mT}$ \\
\hline \hline
\end{tabular}

in the material. The resonance at $g_{\mathrm{db}}=2.0046(3)$ can be assigned to dangling bonds ( $\mathrm{db}$ states) in the disordered regions of the microcrystalline silicon $[35,49,50]$. With the help of its characteristic fingerprint $g$ value, the EDMR line at $g_{\mathrm{h}}=2.0105(5)$ is ascribed to holes in valence band tail states (h centers) in the disordered phase [51,52]. Finally, the slightly asymmetric line at $g_{\mathrm{CE}}=1.9978(3)$ belongs to so-called conduction band tail states (CE states) [48,49,53-55], which are localized in the crystalline phase of the material $[55,56]$. Its asymmetric line profile which is caused by a distribution of $g$ values [48] is considered in the fit.

Besides these well known resonances we find a remaining broad line centered at $g=2.017$ (1) with a line width of 19(1) mT. Both parameters deviate strongly from those mentioned above, which indicates a significantly different structure of the underlying paramagnetic defect. This line will be referred to as TE line. The aim of the following experiments is the identification of this signal and its influence on the photocurrent.

\section{B. Electrical detection of Rabi nutations}

The experimental data described so far suggest that the broad EDMR resonance is connected with a paramagnetic center in a state with $S>1 / 2$. Solid results regarding the spin state of this center can be gained by measuring the Rabi nutations, i.e., the nutations of the spins in the mw $B_{1}$ field. The nutation frequency $\Omega$ allows conclusions about the spin state according to Eq. (1). Rabi traces were measured at three $B_{0}$ positions in the EDMR spectrum as shown in Fig. 4(a). One trace was detected at the position of the CE signal $\left(B_{0}=349.3 \mathrm{mT}\right.$, $\left.g_{\mathrm{CE}}=1.998\right)$ to obtain a reference Rabi frequency for a spin $S=1 / 2$ system. It has previously been shown that spin-dependent hopping transport between two adjacent $\mathrm{CE}$ centers under the given experimental conditions results in an oscillation of the EDMR signal that is dominated by the Rabi frequency of a single $S=1 / 2$ center [38]. Measurements at $336 \mathrm{mT}$ and $356 \mathrm{mT}$ provide the Rabi frequencies of the broad TE signal. The Rabi traces after post-processing and the corresponding frequency spectra are given in Figs. 4(b) and 4(c), respectively.

The Rabi frequency of the $S=1 / 2$ system measured at $g_{\mathrm{CE}}=1.998$ is described by

$$
\Omega_{\mathrm{CE}}=2 \pi v_{\mathrm{CE}}=\gamma_{\mathrm{CE}} B_{1}
$$

following Eq. (1). The experiment yields $v_{\mathrm{CE}}=23.46 \mathrm{MHz}$ at $g_{\mathrm{CE}}=1.998$ so that a mw field strength of $B_{1}=0.84 \mathrm{mT}$ can be calculated. A small frequency contribution at $v \approx$ $47 \mathrm{MHz}$ is observed in the FFT spectrum, which is due to the spin-locking frequency $\Omega_{\mathrm{CE}}^{\mathrm{sl}}=2 \Omega_{\mathrm{CE}}$. This occurs under experimental conditions that allow for a simultaneous manipulation of both spins of a weakly coupled spin pair $[38,57]$. The remaining two traces provide clearly different frequencies of $\nu_{\mathrm{TE}}^{356 \mathrm{mT}}=32.5 \mathrm{MHz}$ and $\nu_{\mathrm{TE}}^{336 \mathrm{mT}}=34.5 \mathrm{MHz}$. Taking the calculated mw field strength $B_{1}$ and the $g$ value $g_{\mathrm{TE}}=2.017$ of the TE line into account, we find that the frequencies appear at approximately $\sqrt{2} \Omega_{\mathrm{CE}}$. The result fits to Rabi frequencies expected for the allowed EPR transitions $\left(m_{\mathrm{S}}=-1 \leftrightarrow m_{\mathrm{S}}=0\right)$ and $\left(m_{\mathrm{S}}=0 \leftrightarrow m_{\mathrm{S}}=+1\right)$ of an $S=1$ system. Hence, the broad EDMR line can be assigned to 

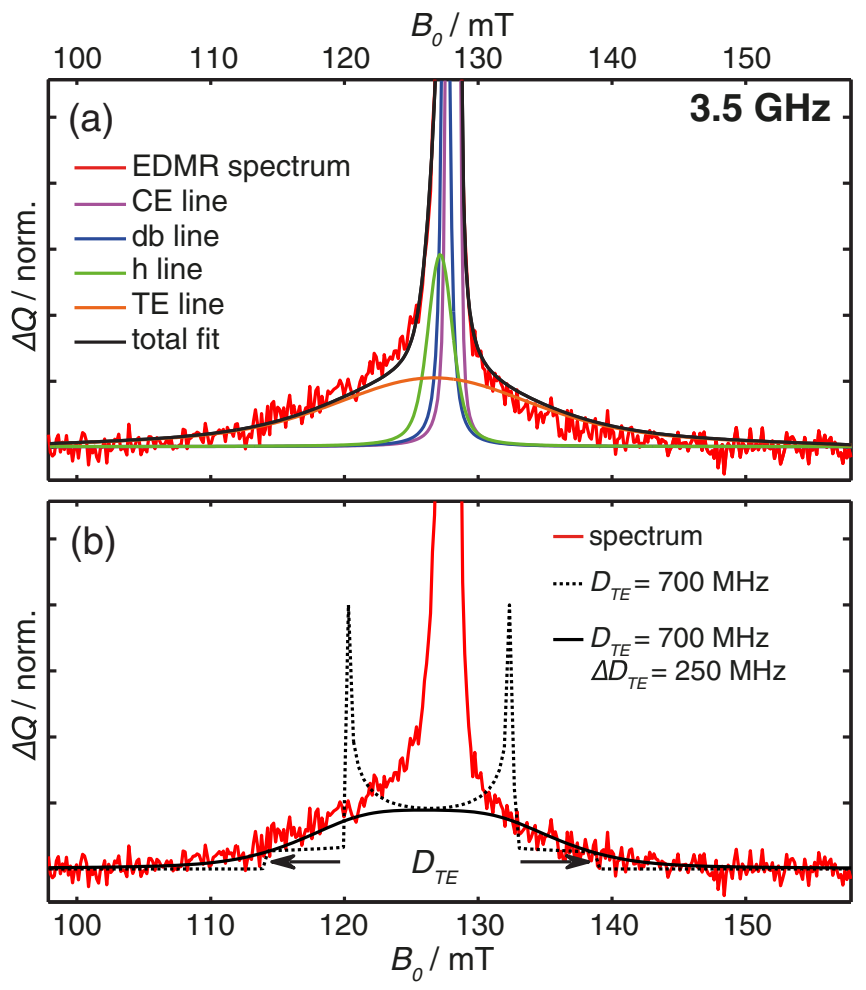

FIG. 5. (a) Field-swept $S$-band EDMR spectrum together with the fit results. The lower $20 \%$ of the signal intensity are shown for better illustration of the TE line. (b) Simulation of dipolar Pake patterns with and without additional broadening (solid and dashed black lines) to describe the $3.5 \mathrm{GHz}$ TE line shape.

paramagnetic centers in the triplet $S=1$ state, which influence the photocurrent. However, the measured frequencies differ from the expected pure $S=1$ frequency at $v_{\mathrm{TE}}=33.54 \mathrm{MHz}$. Such discrepancies can appear when the considered $S=1$ spin is coupled to other spins in the environment as will be discussed later [58,59].

\section{Field-swept EDMR at $S$ band}

In the following section the field-swept EDMR experiment performed at $S$-band frequencies $(3.5 \mathrm{GHz})$ is presented. The $S$-band spectrum together with that measured at $X$ band allow for drawing conclusions about the impact of field-independent line broadening due to the dipolar interaction expected for the triplet TE signal.

The obtained spectrum was evaluated as described in Sec. III A with the only difference that the $g$ values deduced at $9.8 \mathrm{GHz}$ were used as input parameters to fit the $3.5 \mathrm{GHz}$ spectrum. The line widths and line amplitudes were free parameters of the fit. The spectrum and the resulting spectral fit including all four EDMR lines are shown in Fig. 5(a).

The TE signal shows an unusual large width of $\Delta B_{\mathrm{TE}} \approx$ 19(1) $\mathrm{mT}$. It is thus as wide as at $X$-band frequencies despite the fact that the mw frequency is smaller by a factor of 2.8 . This is typical for a line dominated by strong field-independent magnetic interactions like hyperfine or dipolar interaction. However, hyperfine interactions to nuclei in the surrounding as origin of such a strong broadening can be immediately excluded, since the line width of the TE signal is independent of the concentration of the only magnetic nucleus ${ }^{29} \mathrm{Si}$ in the TE state environment (details below in Sec. IIID and Ref. [60]). Hence, the field-independent part of the line width is dominated by the dipolar interaction within the strongly coupled electron spin pair.

In the following, we concentrate on the $3.5 \mathrm{GHz}$ spectrum for the analysis of the dipolar interaction, because at this frequency even the field-dependent width due to Zeeman couplings can be neglected, such that we have access to the full dipolar line width of the TE signal. A resonance line is split under the influence of strong spin-spin coupling by

$$
\Delta B_{\text {split }}=\frac{3}{2} \frac{\mu_{0}}{4 \pi \hbar} \frac{g_{\mathrm{TE}}^{2} \beta_{\mathrm{e}}^{2}}{r^{3}}\left(3 \cos ^{2} \theta-1\right)
$$

in the EDMR spectrum, where $\theta$ represents the angle between the external magnetic field axis and the axis connecting both electron spins. $r$ is the distance of the interacting spins. In powder samples with arbitrary orientations of the spin-spin coupling direction relative to the $B_{0}$ field direction the line doublets add up to a so-called Pake pattern [61]. The outermost edges of this pattern $(\theta=0)$ are split by

$$
D_{\mathrm{TE}}=3 \frac{\mu_{0}}{4 \pi \hbar} \frac{g_{\mathrm{TE}}^{2} \beta_{\mathrm{e}}^{2}}{r^{3}} .
$$

In a first evaluation step we adjust the width $D_{\mathrm{TE}}$ of a Pake pattern by choosing the spin-spin distance $r$ such that it covers the width of the TE line as shown in Fig. 5(b). One can immediately see that the Pake pattern which has pronounced features for $\theta=0$ and $90^{\circ}$ covers the complete field range but does not fit to the broad unstructured TE line. However, when $D_{\mathrm{TE}}$ is assumed to be not constant for all pairs of coupled spins in the sample, but somehow distributed around a mean value, the sharp profile of the Pake pattern could be smeared out. This might be the reason why the triplet signal here shows up as a featureless line.

The influence of a distribution of $D_{\mathrm{TE}}$ values is considered in Fig. 5(b) showing two simulations which are compared to the measured data: (1) a pure $S=1$ Pake pattern with coupling constant $D_{\mathrm{TE}}=700 \mathrm{MHz}$ and (2) the same pattern convolved with a dipolar broadening of $\Delta D_{\mathrm{TE}}=250 \mathrm{MHz}$. $D_{\mathrm{TE}}$ and $\Delta D_{\mathrm{TE}}$ were chosen to reproduce the triplet signal shape despite holding $\Delta D_{\mathrm{TE}}$ as small as possible to avoid unrealistic results. The triplet signal is well described by the simulation, but it is important to note that the given values constitute only an estimation, because many combinations of $D_{\mathrm{TE}}$ and $\Delta D_{\mathrm{TE}}$ lead to good fit results for an unstructured line profile. That is especially true when the $E$ parameter describing the rhombic part of the dipolar coupling is taken into account as well. However, we can connect the obtained $D_{\mathrm{TE}}$ value with an interspin distance $r$ to get an idea of the defect structure. Employing Eq. (4) we can calculate the mean spin-spin distance

$$
r=\left(3 \frac{\mu_{0}}{4 \pi \hbar} \frac{g_{\mathrm{TE}}^{2} \beta_{\mathrm{e}}^{2}}{D_{\mathrm{TE}}}\right)^{1 / 3},
$$

which yields

$$
r / \mathrm{nm}=\left(\frac{38.94 \cdot g_{\mathrm{TE}}^{2}}{D_{\mathrm{TE}} / \mathrm{MHz}}\right)^{1 / 3} \approx 0.6
$$


(a) 2pESEEM

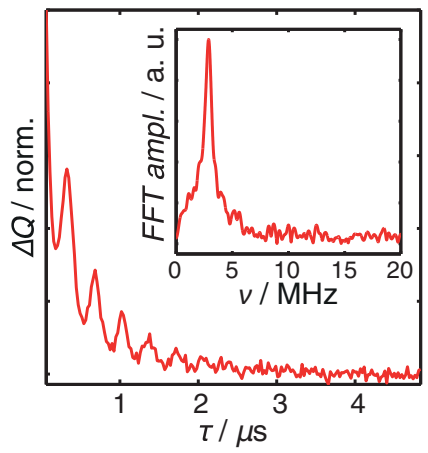

(b) 3pESEEM

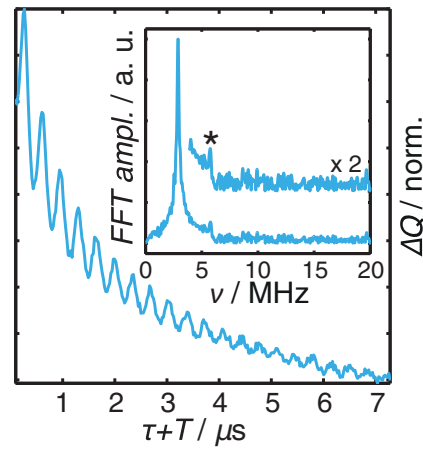

FIG. 6. The 2pESEEM (a) and 3pESEEM (b) time traces detected at $X$ band $\left(B_{0}=340 \mathrm{mT}\right)$ as a function of the interpulse delays $\tau$ and $T+\tau$, respectively. The insets show the corresponding frequency spectra. The asterisk (right inset) indicates a narrow peak at $v=5.77(2) \mathrm{MHz}$.

for $D_{\mathrm{TE}}=700 \mathrm{MHz}$. Including the dipolar broadening $\Delta D_{\mathrm{TE}}$ into the calculation one obtains a range of interspin distances of $0.55-0.7 \mathrm{~nm}$.

Using this broadened TE Pake pattern for the fit of the total EDMR spectrum results in a better fit quality of the marginal areas. However, all deduced resonance positions and widths (cf. Table I) remain unchanged.

\section{Electrically detected ESEEM}

So far, we described an EDMR signal connected with two strongly interacting electron spins in the microcrystalline material. In order to identify the microscopic environment of this triplet state electrically detected two-pulse ESEEM (2pESEEM) [55,62] and three-pulse ESEEM (3pESEEM) [56] experiments were conducted using a $\mu \mathrm{c}-\mathrm{Si}: \mathrm{H}$ solar cell with a ${ }^{29} \mathrm{Si}$-enriched intrinsic layer (sample $\mathrm{C}_{29}$ ). These techniques reveal the nuclei in the vicinity of the paramagnetic species through the hyperfine interaction.

The field position of $B_{0}=340 \mathrm{mT}$ was chosen for the experiments to avoid interferences with other signals. Figure 6(a) displays the $4.9 \mu$ s long $X$-band 2pESEEM trace together with the frequency spectrum. The time trace shows pronounced oscillations. The frequency contributions range between nearly 0 and $6 \mathrm{MHz}$ with a peak at $v_{\mathrm{I}}=2.9 \mathrm{MHz}$, which is the nuclear Larmor frequency of ${ }^{29} \mathrm{Si}$. Frequencies due to other nuclear spins are not observed. Since the limited electron spin phase memory time $T_{\mathrm{M}}$ leads to a broadening of the frequency spectrum, 3pESEEM was employed in the next step [cf. Figs. 3(c) and 6(d)], which circumvents this effect.

The much longer spin-lattice relaxation time $T_{1}$ of the system allows us to record long 3 pESEEM traces. This leads to spectra of increased resolution with frequency contributions at $v_{\mathrm{I}}, v_{ \pm 1}$. The sharp ${ }^{29} \mathrm{Si}$ line at $v_{\mathrm{I}}$ as well as the unstructured contribution between 0 up to $6 \mathrm{MHz}$ are found again. In addition, at 5.77(2) $\mathrm{MHz}$ a small but distinct peak is resolved. It can be assigned to a sharp ${ }^{29} \mathrm{Si}$ double quantum (DQ) peak of the particularly narrow $m_{\mathrm{S}}=0$ transition at $2 v_{0}=5.76 \mathrm{MHz}$, which can be found when two nuclei are simultaneously excited by the mw pulses [63]. An alternative assignment to ${ }^{31} \mathrm{P}$

nuclei $\left(v_{\mathrm{I}}=5.87 \mathrm{MHz}\right.$ at $\left.340 \mathrm{mT}\right)$ from the $n$-doped sample layer is hardly probable due to the frequency discrepancy of $100 \mathrm{kHz}$. Furthermore, ESEEM studies of CE states stemming as well from pure Si environments in the material did not reveal ${ }^{31} \mathrm{P}$ signals [56].

We can conclude that the strongly coupled TE spin pair is located in a pure $\mathrm{Si}$ environment. Neither hydrogen is detected, which would be indicative for disordered regions with passivated dangling bonds, nor ${ }^{31} \mathrm{P}$ or ${ }^{11} \mathrm{~B}$ are found, which would point to signals originating from the doped layers of the solar cell. Consequently, the crystalline Si phase of the material is accommodating the defects responsible for the $\mathrm{TE}$ signal.

In the nuclear frequency range up to $20 \mathrm{MHz}$ studied here, only very small ${ }^{29} \mathrm{Si} \mathrm{HF}$ couplings (maximum $\approx 3 \mathrm{MHz}$ ) were resolved. In addition, comparison of the line widths of the TE signal in EDMR field-swept spectra of the cells $\mathrm{C}_{29}$ and $\mathrm{C}_{28}$ illustrates their independence of the ${ }^{29} \mathrm{Si}$ concentration [60]. This means that HF broadening in the EDMR spectra is negligible and couplings $>20 \mathrm{MHz}$ can be excluded. Both findings indicate a rather extended wave function of the triplet state within the material.

\section{E. Electrically detected ELDOR}

We could show that the TE signal in the EDMR spectrum arises from two strongly coupled spins in the crystalline phase of the microcrystalline silicon solar cell. However, the observation of an EDMR signal after spin manipulation within the triplet manifold alone is in general not feasible. A process is required that allows a triplet-to-singlet conversion via ISC to generate singlet state population which influences the hopping or recombination rate in the cell. Here, SOC can play an important role. Alternatively, coupling to an additional spin which goes along with coupled spin states could promote triplet-to-singlet conversion, as already suggested for other systems $[10,34]$. The additional spin needs to be spatially close to the TE spin to achieve a sufficiently strong interaction. Consequently, spins trapped in CE states could be appropriate candidates, as these states are assigned to the same crystalline silicon phase as the TE spins. Spins in $\mathrm{h}$ and $\mathrm{db}$ states can be discarded, since they are ascribed to the disordered hydrogen-rich regions [50] and are thus too far away.

The electrically detected electron electron double resonance (ED-ELDOR) experiment [10,44] is capable to identify a possible coupling between TE and CE spin species by exploiting the dependence of the EDMR current response on the symmetry of the TE spin-CE spin pair. While the orientation of CE spins is flipped step-by-step by an incrementation of the pulse length $t_{\text {Rabi }}$ (Rabi experiment), the impact of this manipulation on the current response at the resonance position of the TE signal is detected. The peculiarity in the present case is that an $S=1$ couples to an $S=1 / 2$ spin.

Figure 7(a) shows the EDMR spectrum of the ${ }^{29} \mathrm{Si}$-enriched thin-film solar cell $\mathrm{C}_{29}$ used for the ED-ELDOR study. This sample is especially suitable, as the $\mathrm{CE}$ and the TE signals show up with significant intensity, whereas the $h$ and $d b$ resonances are suppressed. This is probably due to the higher crystalline volume fraction of $I_{\mathrm{c}}=67 \%$ and due to substantial ${ }^{29} \mathrm{Si} \mathrm{HF}$ line broadening, which at least affects the db line 

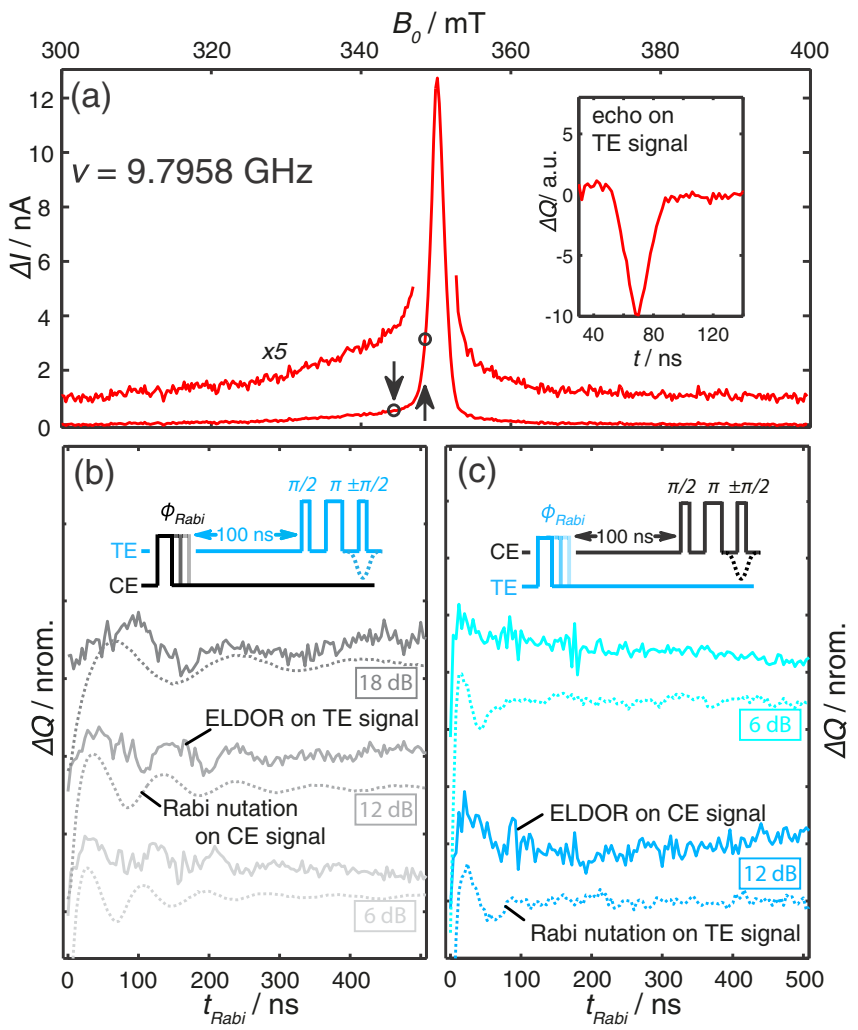

FIG. 7. (a) EDMR spectrum of the ${ }^{29} \mathrm{Si}$-enriched thin-film solar cell used for the ED-ELDOR study. The spectral parts besides the CE resonance are vertically shifted and given with magnified intensity. Spectral positions for spin manipulation are indicated by arrows. Inset: Example of electrically detected spin echo measured on TE signal. For enhancing EDMR signals $(\Delta I / I>0)$ the echo profile is negative. ELDOR traces were recorded on top of this echo. (b) and (c): Modulated part of the ED-ELDOR traces for excitation of Rabi nutations on the CE signal (TE signal) and detection on the TE signal (CE signal), respectively. For comparison Rabi traces (dashed lines) directly detected on the CE signal (TE signal) are shown. The traces are vertically shifted for clarity. The insets show the applied pulse sequences.

[56,64] (further information is given in Ref. [60]). Spins were manipulated on the broad TE signal $(v=9.7958 \mathrm{GHz})$ and on the low field side of the CE resonance $(v=9.6800 \mathrm{GHz})$ within this spectrum by choosing $B_{0}=344.4 \mathrm{mT}$ as indicated by arrows in Fig. 7(a). Both spectral positions are separated by $115.8 \mathrm{MHz}$, which is a compromise between large frequency separation and sufficiently high microwave power (limited by resonator bandwidth) for pulse experiments.

Assuming that CE spins $(S=1 / 2)$ in the sample couple to TE spins $(S=1)$, we expect the highest initial population for those three-spin configurations that have the longest lifetime. By selectively pumping the CE spins within the Rabi experiment the spin configuration of the three-spin system is altered to one with shorter lifetime. This increases the recombination or hopping rate within this system and results in an oscillatory change of the probed TE echo amplitude. This change in time is an imprint of the Rabi nutation with frequency $v_{\mathrm{CE}}$ of the CE signal. The TE echo amplitude is unaffected when no coupling exists.
The ELDOR traces given in Fig. 7(b) indeed show clear oscillations up to $t_{\text {Rabi }} \approx 400 \mathrm{~ns}$ (more details are presented in Ref. [60]). The frequency of these indirectly measured Rabi oscillations decreases with increasing mw power attenuation of the pulse addressing the $\mathrm{CE}$ transition and is identical to the behavior of the corresponding direct CE Rabi experiment (as depicted in Sec. III B). Hence, we conclude to see the CE Rabi frequency $v_{\mathrm{CE}}$ on the TE spin echo. Oscillations due to TE Rabi frequencies $\approx \sqrt{2} v_{\mathrm{CE}}$ or due to off-resonant excitations can be discarded [60]. This dependence of the TE echo intensity on the CE spin orientation means that both the CE $S=1 / 2$ and the TE $S=1$ are coupled and take part in a combined spin-dependent process affecting the sample conductivity.

This experiment is repeated in the reverse direction to crosscheck the result. Therefore, the Rabi pulse was applied on the TE transition and the final spin pair state was read out on the CE transition as shown in Fig. 7(c). Both the ELDOR and the directly measured Rabi traces show a strongly damped oscillation of comparable oscillation period despite the small signal-to-noise ratio. This means the ELDOR signal detected on the $\mathrm{CE}$ resonance shows the expected triplet Rabi nutation frequency $\approx \sqrt{2} v_{\mathrm{CE}}$.

In all ELDOR traces one observes a modulation depth $K \ll 1$, [60] since on one hand only small parts of the EDMR lines could be excited and on the other hand competitive transport processes, e.g., hopping via $\mathrm{CE}$ states give rise to contributions unaffected by the ELDOR pump pulse.

\section{DISCUSSION}

\section{A. Structural origin of CE and TE states}

The crystalline phase of microcrystalline silicon accounts for $\approx 50-70 \%$ of the cell material and is characterized by agglomerations of crystallites separated by thin irregular interspaces [cf. Fig. 8(a)]. This space contains dangling bonds [50]. However, also the microcrystals exhibit a pronounced substructure connected with point and extended defects instead of a perfect crystal structure [65-67]. These sites are potential candidates for accommodating the observed CE or TE states. In the following, we discuss point as well as extended defect types and whether their magnetic properties fit to those found for the $\mathrm{CE}$ and $\mathrm{TE}$ states.

The signal of conduction band tail (CE) states is one of the most studied EPR signals of microcrystalline silicon and was assigned early on to conduction electrons in the crystalline zones [54,68,69]. Electrically detected ESEEM measurements confirmed this by assigning them to a pure silicon environment free of doping atoms and hydrogen [55]. However, the structural origin of CE states within the crystallites remained a point of discussion. Recently, ${ }^{29} \mathrm{Si} \mathrm{HF}$ studies were presented for the CE EDMR signal showing that the CE signal is dominated by small ${ }^{29} \mathrm{Si} \mathrm{HF}$ coupling constants indicative for a rather delocalized wave function. HF interactions to other nuclei were not observed [56].

Point defects in undoped crystalline silicon like vacancies or interstitials are possible CE state locations and occur in a large variety of different defect structures [8,70,71]. However, these defects are in general characterized by localized wave functions. Such a compact spin density leads to large HF 


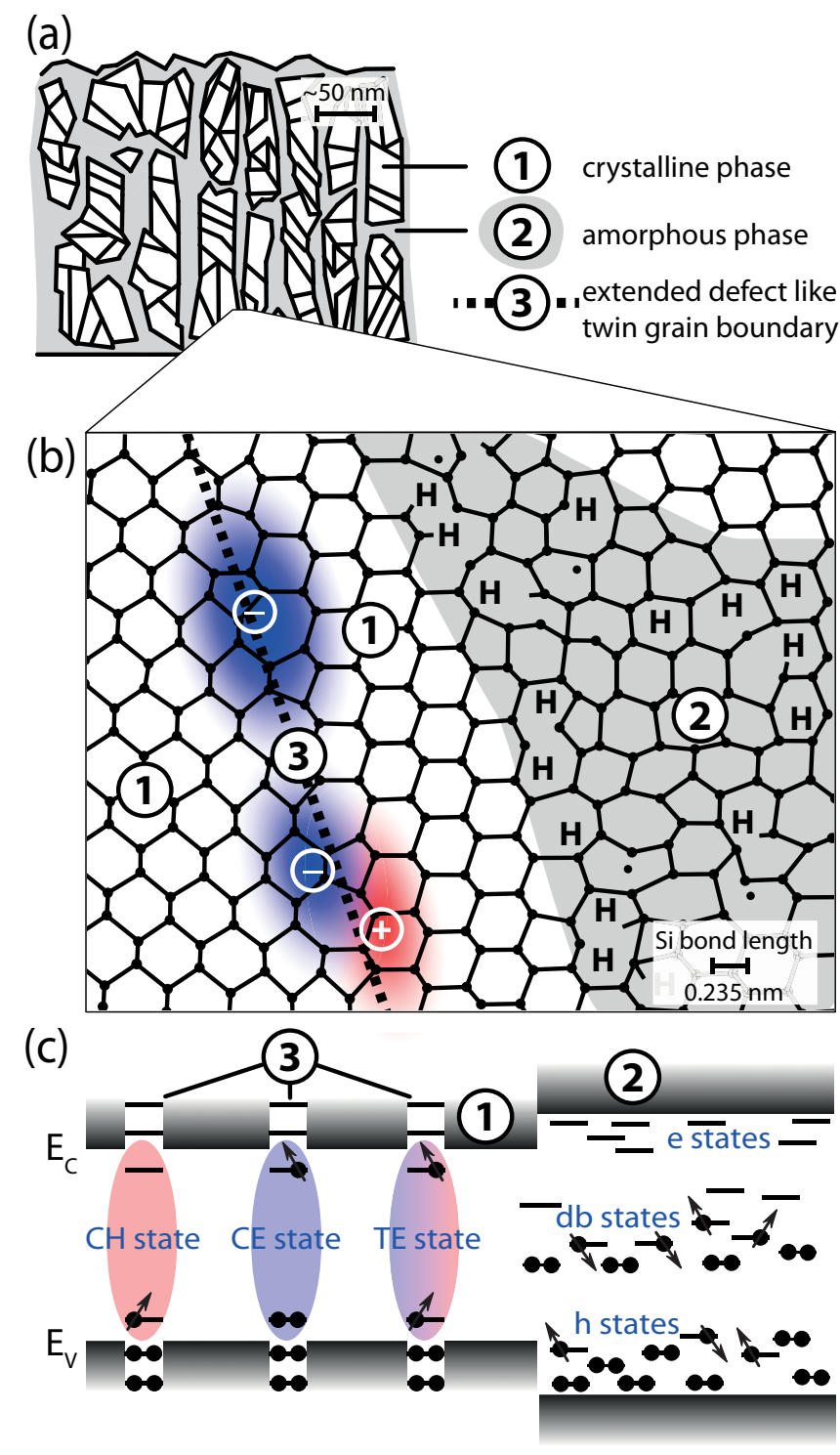

FIG. 8. Schematic material and energetic structure of microcrystalline silicon. (a) Cross section of a $\mu \mathrm{c}-\mathrm{Si}: \mathrm{H}$ layer showing crystallites (1) embedded in an amorphous matrix (2). (b) Schematic top view of the nanometer-scale structure of microcrystalline silicon. Within the crystallites extended defects (3) like stacking faults and twin grain boundaries are found. The dashed line indicates a grain boundary between two crystals of different orientation. At these defects photoexcited charges (illustrated by the red and blue clouds) can be trapped and give rise to CE and TE EDMR signals. The gray shaded zone constitutes a hydrogen-rich amorphous region with dangling bonds $(\bullet$ ) and passivating protons (H). (c) Schematic energy band structure for a spatial cut across grain boundaries and an amorphous zone according to (b). In the crystalline regions TE, CE, and $\mathrm{CH}$ states are located at extended defects, whereas the amorphous zones accommodate $\mathrm{e}, \mathrm{h}$, and $\mathrm{db}$ states. In both sketches relative distances and dimensions of material regions, defects, and interfaces are modified for reasons of clarity.

couplings to ${ }^{29} \mathrm{Si}$ nuclei (in the $>100 \mathrm{MHz}$ range) $[8,9,72]$, which is not consistent with our observations [56]. We therefore rule out such point defects as the site for the $\mathrm{CE}$ state location.
However, microcrystalline silicon accommodates, beyond the point defects known from bulk crystals, a large number of spatially extended defects such as stacking faults or twin grain boundaries [65-67] [cf. the sketch in Fig. 8(b)]. These extended defects can act as traps for photoexcited states. Many of these defects come along without broken bonds [73,74]. Strained Si bonds within these extended defect regions might induce energy levels within the band gap which can act as traps for charge carriers and excitons. Their linear or planar defect character could lead to an extended defect spin density resulting in small HF coupling constants. This is in full agreement with the experimental results and indicative for a localization of the CE states on these extended defects within silicon crystallites.

In parallel to this, the EDMR TE signal is, as well, characterized by weak HF coupling constants to surrounding ${ }^{29} \mathrm{Si}$ nuclei. Additionally, no HF signals of other nuclei were found. This leads us to the same conclusion that TE states reside at extended defects. This is supported by the TE line width, which is relatively small compared to widths of triplet signals in other disordered Si-based materials [15] and therefore indicates a larger mean spin-spin distance of the strongly coupled spin pair and, hence, a more extended defect character. Finally, the assignment of CE and TE states to the same defect type is corroborated by our ED-EDLDOR results, which reveal a mutual coupling between CE and TE spins, such that they have to be in close proximity with respect to each other.

\section{B. Energetic structure of CE and TE states and spin-dependent transport processes}

In the previous section, we assigned the $\mathrm{CE}$ as well as the $\mathrm{TE}$ states to extended defects in the crystalline regions based on the results of the different EDMR techniques. In the following we discuss how these states can be excited and how they are involved in spin-dependent transport processes in the cell.

In Fig. 8(c) the schematic picture of the $\mu \mathrm{c}-\mathrm{Si}: \mathrm{H}$ energy structure is shown as a function of the position in the lattice for a spatial cut across extended defects in the crystalline regions according to Fig. 8(b). Extended defects are expected to induce shallow states close to the band edges rather than deep levels. Indeed, calculations on the energetic structure of stacking faults and grain boundaries show that the bond distortions are large enough to allow for shallow states close to the energy bands [74-78]. Hence, we consider extended defects as shallow quantum wells embedded in a crystalline energy environment, an established approach for the description of tail states in disordered silicon $[68,79,80]$. This well structure leads to spatially localized states with an energy distribution extending into the band gap as sketched in Fig. 8(c).

Without photoexcitation and at low temperatures the states associated with the valence band (valence band tail states) are filled, whereas the states related to the conduction band (conduction band tail states) are empty. Photoexcitation of electrons from valence band tail states to conduction band tail states generates geminate electron-hole pairs, which are bound by the Coulomb interaction. These pairs are excitons created in the singlet state $|S\rangle$. However, fast ISC leads to the population of the triplet state comprising the sublevels $\left|T_{ \pm}\right\rangle$and 
$\left|T_{0}\right\rangle$. Remaining singlet excitons almost immediately relax by charge carrier recombination, whereas the triplet excitons are long lived because the $\left|T_{\mathrm{i}}\right\rangle \rightarrow|S\rangle$ transitions to the ground state $|S\rangle$ are only weakly allowed by SOC [9,33,81]. This results in a high equilibrium population of triplet excitons in the material which is accessible to EPR.

To affect the sample conductivity in the solar cell a charge carrier process is necessary after the spin manipulation of the triplet TE state sublevel population. Otherwise no EDMR signal is observed. We assume that a triplet exciton is involved in a charge carrier recombination process together with a $\mathrm{CE}$ spin close by. The excess energy $\Delta E$ released in the recombination step of the exciton is transferred to the spin trapped in a CE state in an Auger-like process $[10,82]$ comparable to Auger recombination of donor-bound excitons in silicon [83,84]. Thereby the CE spin is excited to the conduction band, where it contributes to the sample conductivity in the form of a positive current response in the EDMR spectrum.

To allow this Auger recombination process two conditions have to be met regarding the three-spin system consisting of the triplet exciton in state $\left|T_{\mathrm{i}}\right\rangle$ and the CE doublet in state $\left|D_{\mathrm{i}}\right\rangle$. On one hand, both participating spins have to be in close proximity for the Auger energy transfer. This proximity leads to dipolar and exchange coupling between both spins. On the other hand, the triplet exciton must be converted into a singlet exciton. Otherwise the exciton recombination is not probable. For this, we consider spin states of the coupled three-spin system in the triplet-doublet basis

$$
\begin{aligned}
|1\rangle & =\left|T_{+}, \uparrow\right\rangle \\
|2\rangle & =\cos \theta\left|T_{0}, \uparrow\right\rangle+\sin \theta\left|T_{+}, \downarrow\right\rangle \\
|3\rangle & =\cos \phi\left|T_{-}, \uparrow\right\rangle-\sin \phi\left|T_{0}, \downarrow\right\rangle \\
|4\rangle & =\cos \theta\left|T_{+}, \downarrow\right\rangle-\sin \theta\left|T_{0}, \uparrow\right\rangle \\
|5\rangle & =\cos \phi\left|T_{0}, \downarrow\right\rangle+\sin \phi\left|T_{-}, \uparrow\right\rangle \\
|6\rangle & =\left|T_{-}, \downarrow\right\rangle,
\end{aligned}
$$

with $\left|T_{\mathrm{i}}, D_{\mathrm{i}}\right\rangle$ the uncoupled product states of both spins and $\theta$, $\phi$ being the mixing angles according to Ref. [59]. The triplet and doublet spins in the three-spin-system are not strongly coupled $(\theta, \phi$ rather small), because the EDMR resonances of the triplet exciton and the CE spin appear as individual lines (cf. Sec. III A). Furthermore, the Rabi experiment clearly reveals doublet and triplet frequencies (cf. Sec. III B). However, a discrepancy of approximately $1 \mathrm{MHz}$ was found between the detected and the expected TE Rabi frequency, which can be explained by the influence of the additional doublet spin $S=1 / 2$ coupling to the triplet spin $S=1$. The exchange or dipolar coupling leads to a shift of the Rabi frequencies of both triplet transitions, which can be analytically described [59]. Coupling constants in the range of $20-80 \mathrm{MHz}$ can be estimated based on the present Rabi results, but additional experiments are necessary for more detailed information about the relative impact of exchange or dipolar coupling on the total coupling strength. The shift of the Rabi frequency is only observed on the TE signal and not on the CE signal, since the latter arises mostly from hopping processes between weakly coupled spins in CE states yielding the pure $S=1 / 2$ Rabi frequency.

Summarizing, we find an intermediately coupled tripletdoublet spin system. Both spins can still be manipulated individually, but the mutual coupling leads to a mixing of the uncoupled product states according to Eq. (7).

Before the spin manipulation the highest population is assumed to be found in those states $|i\rangle$ with the longest lifetimes, which, in turn, are largely dependent on the ISC driven relaxation probability of every individual triplet state $\left|T_{\mathrm{i}}\right\rangle$. Although details are missing about the underlying processes that increase triplet exciton relaxation probabilities, further conclusions about the spin-dependent processes of the three-spin system are possible. On one hand, inducing a triplet spin transition $\left|T_{ \pm}\right\rangle \leftrightarrow\left|T_{0}\right\rangle$ by a mw $\pi$-pulse converts the initial long-lived spin configuration $|l\rangle$ into a short-lived one $|s\rangle$ going along with an increased probability of singlet exciton generation. Subsequently, the Auger recombination process can take place and the CE spin in state $\left|D_{\mathrm{i}}\right\rangle$ is excited into the conduction band. An EDMR current response is detected at the resonance position of the TE signal. On the other hand, inducing a CE spin transition $|\uparrow\rangle \leftrightarrow|\downarrow\rangle$ should in general not alter the spin state of the triplet exciton. However, the coupling between the triplet exciton $\left|T_{\mathrm{i}}\right\rangle$ and the $\mathrm{CE}$ spin $\left|D_{\mathrm{i}}\right\rangle$ leads to a mixture of the uncoupled product states $\left|T_{\mathrm{i}}, D_{\mathrm{i}}\right\rangle$ characterized by individual lifetimes as shown in Eq. (7). This state mixing allows spin flip-flop processes [85] within the triplet-doublet system, which finally gives rise to a population of short-lived pair states. Thus, the exciton is, again, able to recombine and a current response upon the spin manipulation of the doublet $\left|D_{\mathrm{i}}\right\rangle$ states is detected at the CE resonance position.

Support for this spin-dependent recombination process within the triplet-doublet system comes from ODMR studies of microcrystalline silicon [21,22]. The ODMR spectra measured at luminescence energies of $0.75 \mathrm{eV}$, indeed, reveal two enhancing signals at $g=1.9997(\Delta B=1.85 \mathrm{mT})$ and $g=$ $2.016(\Delta B=10 \mathrm{mT})$ similar to the $\mathrm{CE}$ and $\mathrm{TE}$ line parameters of the present study. Here, the excess energy is probably emitted in the form of a photon giving rise to photoluminescence observable in ODMR experiments. The increase of luminescence means here that the spin manipulation increases the probability of radiative exciton recombination in the material.

Finally, we come back to the energetic structure of $\mu \mathrm{c}-\mathrm{Si}: \mathrm{H}$ as displayed in Fig. 8(c). Here, we assigned the TE triplet states to photoexcited electron-hole pairs trapped at extended defects. In addition, we can interpret electrons in conduction band tail (CE) states as the negative charge state of extended defects as sketched in the schematic energy structure. This is consistent with the common picture of CE states being localized states in the $\mathrm{c}-\mathrm{Si}$ band gap in energetic proximity to the conduction band [86], which are observed in EPR and EDMR spectra of microcrystalline silicon under illumination or negative doping $[50,86]$. On the other hand, holes trapped in states close to the valence band (referred to as $\mathrm{CH}$ states [49]) correspond to the positive charge state of extended defects. However, these hole states are hard to detect, probably due to strong line broadening induced by random local lattice strain comparable to the broadening effects on EPR hole signals in crystalline silicon $[87,88]$. The correlation of TE, CE, and $\mathrm{CH}$ 
states with extended defects in the crystalline phase of $\mu \mathrm{c}-\mathrm{Si}: \mathrm{H}$ shows that these defects act as traps for charge carriers and can influence the material conductivity at low $T$.

It is likely that the same processes are active at room temperature as well, but may not be detectable by EDMR techniques due to faster spin-relaxation rates and/or shorter triplet exciton lifetimes at $T=300 \mathrm{~K}$. This is, for instance, the case for triplet exciton generation via back-electron transfer, which occurs in many absorber materials used in solar cells made from organic semiconductors. These processes have equally been studied using EPR techniques at low temperatures [89] and their relevance for room temperature transport has initially been questioned. However, ultrafast optical techniques have confirmed that the same processes are active at room temperature as well [1]. We believe that the same is true for loss mechanisms involving triplet excitons in thin-film silicon materials, namely that the processes probed at low temperatures may also be relevant for room-temperature transport. It remains interesting to see whether ultrafast optical data will corroborate the EDMR findings also for this material system.

From the amorphous phase of microcrystalline silicon only the signals of dangling bonds and valence band tail states are observed (cf. Sec. III A). Signals of a-Si:H conduction band tail (e) states or phosphorus donor (P) states are absent, although they are well known from intrinsic and $n$-doped a$\mathrm{Si}: \mathrm{H}$ samples. Based on our model this might be related to the large a-Si:H mobility gap compared to the small c-Si energy gap and with the energy band offsets, such that transport via $\mathrm{h}$ states is possible, whereas transport via e or $\mathrm{P}$ states is suppressed. The energetic position of e and $\mathrm{P}$ states above the c-Si conduction band edge could lead to their depopulation at low temperature, which would explain why the e and P signals are neither measured by EPR nor by EDMR in microcrystalline silicon [35,50] but clearly visible in amorphous silicon [48]. The deeper understanding of this aspect, however, requires more details about energy band bending and offsets between a-Si:H and c-Si regions in microcrystalline silicon.

\section{SUMMARY AND OUTLOOK}

We presented a comprehensive EDMR study applied to state-of-the-art microcrystalline silicon solar cells with the focus on the TE signal arising from long-lived triplet excitons trapped at extended defects within Si crystallites. The excitons can couple to spins in shallow CE states and take part in a mutual spin-dependent recombination process. The energy structure of the extended defects can be described by quantum wells embedded in the c-Si energy bands giving rise to energy levels in proximity to the conduction and valence band edges better known as conduction (CE) and valence $(\mathrm{CH})$ band tail states. The observed EDMR signals from the crystalline and amorphous regions could be associated with spin-dependent transitions between localized defect states found in microcrystalline silicon.

The EDMR methodology we have used to study triplet states in inorganic disordered semiconductors can also be employed to probe spin-dependent processes involving triplet excitons in organic materials. Due to the low dielectric constant of organic semiconductors, which translates into large exciton binding energies, triplet excitons can substantially influence the electrical and optical properties of this class of materials. It was recently shown that triplet excitons in polymer:fullerene bulk heterojunction solar cells indeed give rise to EDMR signals [90]. The approach presented here can thus be used to identify and elucidate EDMR-active processes in organic solar cells. It can further help to explore the microscopic environment of triplet excitons that influence the photocurrent.

\section{ACKNOWLEDGMENTS}

We are grateful to K.-P. Dinse (FU Berlin) for helpful discussions and appreciate financial support from BMBF (EPR-Solar network project 03SF0328), DFG (SPP 1601) and the Helmholtz Association (Energie-Allianz HybridPhotovoltaik).
[1] A. Rao, P. C. Y. Chow, S. Gelinas, C. W. Schlenker, C. Z. Li, H. L. Yip, A. K. Y. Jen, D. S. Ginger, and R. H. Friend, Nature (London) 500, 435 (2013).

[2] A. Distler, P. Kutka, T. Sauermann, H. J. Egelhaaf, D. M. Guldi, D. D. Nuzzo, S. C. J. Meskers, and R. A. J. Janssen, Chem. Mater. 24, 4397 (2012).

[3] A. Köhler and H. Bässler, Mater. Sci. Eng., R 66, 71 (2009).

[4] F. Etzold, I. A. Howard, N. Forler, D. M. Cho, M. Meister, H. Mangold, J. Shu, M. R. Hansen, K. Mullen, and F. Laquai, J. Am. Chem. Soc. 134, 10569 (2012).

[5] T. F. Schulze and T. W. Schmidt, Energy Environ. Sci. 8, 103 (2015).

[6] M. C. Hanna and A. J. Nozik, J. Appl. Phys. 100, 074510 (2006).

[7] W. Shockley and H. J. Queisser, J. Appl. Phys. 32, 510 (1961).

[8] K. L. Brower, Phys. Rev. B 4, 1968 (1971).

[9] L. S. Vlasenko, Y. V. Martynov, T. Gregorkiewicz, and C. A. J. Ammerlaan, Phys. Rev. B 52, 1144 (1995).

[10] D. P. Franke, F. Hoehne, L. S. Vlasenko, K. M. Itoh, and M. S. Brandt, Phys. Rev. B 89, 195207 (2014).
[11] R. A. Street, Hydrogenated amorphous silicon (Cambridge University Press, New York, 2005).

[12] T. Aoki, T. Shimizu, S. Komedoori, S. Kobayashi, and K. Shimakawa, J. Non-Cryst. Solids 338-340, 456 (2004).

[13] T. Aoki, T. Shimizu, D. Saito, and K. Ikeda, J. Optoelectron. Adv. Mats. 7, 137 (2005).

[14] M. S. Brandt and M. Stutzmann, Appl. Phys. Lett. 58, 1620 (1991).

[15] M. S. Brandt and M. Stutzmann, Solid State Commun. 93, 473 (1995).

[16] S. Y. Lee, S. Y. Paik, D. R. McCamey, J. Hu, F. Zhu, A. Madan, and C. Boehme, Appl. Phys. Lett. 97, 192104 (2010).

[17] J. Meier, R. Flückiger, H. Keppner, and A. Shah, Appl. Phys. Lett. 65, 860 (1994).

[18] O. Vetterl, F. Finger, R. Carius, P. Hapke, L. Houben, O. Kluth, A. Lambertz, A. Muck, B. Rech, and H. Wagner, Sol. Energ. Mat. Sol. Cells 62, 97 (2000).

[19] V. Smirnov, A. Lambertz, B. Grootoonk, R. Carius, and F. Finger, J. Non-Cryst. Solids 358, 1954 (2012). 
[20] F. Meillaud, A. Billet, C. Battaglia, M. Boccard, G. Bugnon, P. Cuony, M. Charrière, M. Despeisse, L. Ding, JordiEscarrePalou, S. Hänni, L. Löfgren, S. Nicolay, G. Parascandolo, M. E. Stckelberger, and C. Ballif, IEEE J. Photovolt. 2, 236 (2012).

[21] F. Boulitrop and A. Chenevas-Paule, J. Phys. (France) 43, 153 (1982).

[22] F. Boulitrop, A. Chenevas-Paule, and D. J. Dunstan, Solid State Commun. 48, 181 (1983).

[23] W. Böttler, V. Smirnov, J. Hupkes, and F. Finger, Phys. Status Solidi A 209, 1144 (2012).

[24] D. Kaplan, I. Solomon, and N. F. Mott, J. Phys. Lett. 39, L51 (1978).

[25] M. Stutzmann, M. S. Brandt, and M. W. Bayerl, J. Non-Cryst. Solids 266-269, 1 (2000).

[26] C. Boehme and K. Lips, Phys. Rev. B 68, 245105 (2003).

[27] A. R. Stegner, C. Boehme, H. Huebl, M. Stutzmann, K. Lips, and M. S. Brandt, Nat. Phys. 2, 835 (2006).

[28] M. S. Brandt and M. Stutzmann, Phys. Rev. B 43, 5184 (1991).

[29] G. Kawachi, C. F. O. Graeff, M. S. Brandt, and M. Stutzmann, Phys. Rev. B 54, 7957 (1996).

[30] C. Boehme and K. Lips, in Charge Transport in Disordered Solids with Applications in Electronics, edited by S. Baranovski (Wiley, Chichester, England; Hoboken, NJ, 2006), pp. 179-219.

[31] U. E. Steiner and T. Ulrich, Chem. Rev. 89, 51 (1989).

[32] L. Liu, Phys. Rev. 126, 1317 (1962).

[33] S. K. Lower and M. A. El-Sayed, Chem. Rev. 66, 199 (1966).

[34] R. L. Vranch, B. Henderson, and M. Pepper, Appl. Phys. Lett. 53, 1299 (1988).

[35] J. Behrends, A. Schnegg, M. Fehr, A. Lambertz, S. Haas, F. Finger, B. Rech, and K. Lips, Philos. Mag. 89, 2655 (2009).

[36] C. Boehme and K. Lips, Appl. Phys. Lett. 79, 4363 (2001).

[37] C. Boehme and K. Lips, Physica B (Amsterdam) 376-377, 930 (2006).

[38] C. Meier, J. Behrends, and R. Bittl, Mol. Phys. 111, 2683 (2013).

[39] A. Schweiger and G. Jeschke, Principles of Pulse Electron Paramagnetic Resonance (Oxford University Press, New York, 2001).

[40] J. Harmer, G. Mitrikas, A. Schweiger, in High Resolution EPR, Biological Magnetic Resonance, Vol. 28, edited by G. Hanson and L. Berliner (Springer Science+Business Media, New York, 2009), Chap. 2, pp. 13-61.

[41] H. Huebl, F. Hoehne, B. Grolik, A. R. Stegner, M. Stutzmann, and M. S. Brandt, Phys. Rev. Lett. 100, 177602 (2008).

[42] D. R. McCamey, J. Van Tol, G. W. Morley, and C. Boehme, Science 330, 1652 (2010).

[43] F. Hoehne, L. Dreher, H. Huebl, M. Stutzmann, and M. S. Brandt, Phys. Rev. Lett. 106, 187601 (2011).

[44] F. Hoehne, H. Huebl, B. Galler, M. Stutzmann, and M. S. Brandt, Phys. Rev. Lett. 104, 046402 (2010).

[45] R. E. Martin, M. Pannier, F. Diederich, V. Gramlich, M. Hubrich, and H. W. Spiess, Angew. Chem., Int. Ed. Engl. 37, 2833 (1998).

[46] S. Stoll and A. Schweiger, J. Magn. Reson. 178, 42 (2006).

[47] J. Behrends, A. Schnegg, C. Boehme, S. Haas, H. Stiebig, F. Finger, B. Rech, and K. Lips, J. Non-Cryst. Solids 354, 2411 (2008).

[48] C. Meier, J. Behrends, C. Teutloff, O. Astakhov, A. Schnegg, K. Lips, and R. Bittl, J. Magn. Reson. 234, 1 (2013).

[49] K. Lips, P. Kanschat, and W. Fuhs, Sol. Energ. Mat. Sol. Cells 78, 513 (2003).
[50] F. Finger, J. Müller, C. Malten, and H. Wagner, Philos. Mag. B 77, 805 (1998).

[51] K. Lips, S. Schütte, and W. Fuhs, Philos. Mag. B 65, 945 (1992).

[52] H. Dersch, J. Stuke, and J. Beichler, Phys. Status Solidi B $\mathbf{1 0 5}$ 265 (1981).

[53] J. Müller, F. Finger, R. Carius, and H. Wagner, Phys. Rev. B 60, 11666 (1999)

[54] F. Finger, C. Malten, P. Hapke, R. Carius, R. Flückiger, and H. Wagner, Philos. Mag. Lett. 70, 247 (1994).

[55] M. Fehr, J. Behrends, S. Haas, B. Rech, K. Lips, and A. Schnegg, Phys. Rev. B 84, 193202 (2011).

[56] C. Meier, C. Teutloff, O. Astakhov, F. Finger, R. Bittl, and J. Behrends, Appl. Magn. Reson. 45, 1075 (2014).

[57] Y. N. Molin, O. A. Anisimov, V. M. Grigoryants, V. K Molchanov, and K. M. Salikhov, J. Phys. Chem. 84, 1853 (1980).

[58] K. Ayabe, K. Sato, S. Nakazawa, S. Nishida, K. Sugisaki, T. Ise, Y. Morita, K. Toyota, D. Shiomi, M. Kitagawa, S. Suzuki, K. Okada, and T. Takui, Mol. Phys. 111, 2767 (2013).

[59] T. L. Keevers, W. J. Baker, and D. R. McCamey, Phys. Rev. B 91, 205206 (2015).

[60] See Supplemental Material at http://link.aps.org/supplemental/ 10.1103/PhysRevB.94.045302 for additional experimental and theoretical details.

[61] G. E. Pake, J. Chem. Phys. 16, 327 (1948).

[62] F. Hoehne, J. Lu, A. R. Stegner, M. Stutzmann, M. S. Brandt, M. Rohrmüller, W. G. Schmidt, and U. Gerstmann, Phys. Rev. Lett. 106, 196101 (2011).

[63] L. Liesum and A. Schweiger, J. Chem. Phys. 114, 9478 (2001).

[64] D. K. Biegelsen and M. Stutzmann, Phys. Rev. B 33, 3006 (1986).

[65] L. Houben, M. Luysberg, and R. Carius, Phys. Rev. B 67, 045312 (2003).

[66] L. Houben, M. Luysberg, P. Hapke, R. Carius, F. Finger, and H. Wagner, Philos. Mag. A 77, 1447 (1998).

[67] M. Luysberg, P. Hapke, R. Carius, and F. Finger, Philos. Mag. A 75, 31 (1997).

[68] M. Taniguchi, M. Hirose, Y. Osaka, S. Hasegawa, and T. Shimizu, Jpn. J. Appl. Phys. 19, 665 (1980).

[69] S. Hasegawa, S. Narikawa, and Y. Kurata, Philos. Mag. B 48, 431 (1983).

[70] E. G. Sieverts, Phys. Status Solidi B 120, 11 (1983).

[71] Y. H. Lee, N. N. Gerasimenko, and J. W. Corbett, Phys. Rev. B 14, 4506 (1976).

[72] W. M. Chen, O. O. Awadelkarim, B. Monemar, J. L. Lindström, and G. S. Oehrlein, Phys. Rev. Lett. 64, 3042 (1990).

[73] J. Hornstra, J. Phys. Chem. Solids 5, 129 (1958).

[74] M. Kohyama, Modell. Simul. Mater. Sci. Eng. 10, R31 (2002).

[75] C. Raffy, J. Furthmüller, and F. Bechstedt, J. Phys. Condens. Matter 14, 12725 (2002).

[76] M. Y. Chou, M. L. Cohen, and S. G. Louie, Phys. Rev. B 32, 7979 (1985).

[77] D. P. DiVincenzo, O. L. Alerhand, M. Schlüter, and J. W. Wilkins, Phys. Rev. Lett. 56, 1925 (1986).

[78] R. E. Thomson and D. J. Chadi, Phys. Rev. B 29, 889 (1984).

[79] M. H. Brodsky, Solid State Commun. 36, 55 (1980).

[80] P. W. Anderson, Phys. Rev. 109, 1492 (1958).

[81] A. M. Frens, M. E. Braat, A. B. van Oosten, and J. Schmidt, Mater. Sci. Forum 117-118, 195 (1993). 
[82] W. J. Baker, D. R. McCamey, K. J. van Schooten, J. M. Lupton, and C. Boehme, Phys. Rev. B 84, 165205 (2011).

[83] D. F. Nelson, J. D. Cuthbert, P. J. Dean, and D. G. Thomas, Phys. Rev. Lett. 17, 1262 (1966).

[84] W. Schmid, Phys. Status Solidi B 84, 529 (1977).

[85] A. Abragam, Principles of Nuclear Magnetism (Oxford University Press Inc., New York, 2011).

[86] M. Kondo, T. Nishimiya, K. Saito, and A. Matsuda, J. NonCryst. Solids 227-230, 1031 (1998).
[87] G. Feher, J. C. Hensel, and E. A. Gere, Phys. Rev. Lett. 5, 309 (1960).

[88] A. R. Stegner, H. Tezuka, T. Andlauer, M. Stutzmann, M. L. W. Thewalt, M. S. Brandt, and K. M. Itoh, Phys. Rev. B 82, 115213 (2010).

[89] L. Franco, A. Toffoletti, M. Ruzzi, L. Montanari, C. Carati, L. Bonoldi, and R. Po, J. Phys. Chem. C 117, 1554 (2013).

[90] B. Z. Tedlla, F. Zhu, M. Cox, J. Drijkoningen, J. Manca, B. Koopmans, and E. Goovaerts, Adv. Energy Mater. 5, 1401109 (2015). 